\title{
Cut-off points of waist circumference and body mass index for detecting diabetes, hypercholesterolemia and hypertension according to National Non-Communicable Disease Risk Factors Surveillance in Iran
}

\author{
Abbaszadeh-Ahranjani Shabnam¹, Kashani Homa², Mohajeri-Tehrani Mohammad Reza', \\ Larijani Bagher ${ }^{1}$, Forouzanfar Mohammad Hossein ${ }^{1,2}$, Afshani Hamidreza ${ }^{3}$
}

\author{
1Endocrinology and Metabolism Research Center, Tehran University of Medical \\ Sciences, Iran \\ 2Department of Epidemiology and Biostatistics, School of Public Health, Tehran \\ University of Medical Sciences, Iran \\ 3 Iranian Society of Endocrinology, Iran
}

Submitted: 11 July 2011

Accepted: 25 October 2011

Arch Med Sci 2012; 8, 4: 614-621

DOI: 10.5114/aoms.2012.30284

Copyright $\odot 2012$ Termedia \& Banach

\section{Abstract}

Introduction: The cut-off points of waist circumference and body mass index (BMI) are varied according to different races. There is a dearth of information on these indices especially in Iranian adults. We sought to estimate the cut-off points of waist circumference and BMI for detecting diabetes, hypercholesterolemia, and hypertension.

Material and methods: The data were gathered by the First Iranian Non-Communicable Disease Survey in 2005. In total, 70,981 participants between 25 and 64 years of old were selected via random multistage cluster sampling. Receiver operating characteristic curves were used to show the cut-off points of waist circumference and BMI for detecting diabetes, hypercholesterolemia, and hypertension. The maximum value the sum of sensitivity and specificity indicated the cut-off point.

Results: The cut-off points of waist circumference according to maximum sum of sensitivity and specificity for detecting hypertension, diabetes, and hypercholesterolemia in men were $89.7 \mathrm{~cm}, 89.4 \mathrm{~cm}$ and $88.2 \mathrm{~cm}$ and in women were $93.9 \mathrm{~cm}, 96.2 \mathrm{~cm}$ and $90 \mathrm{~cm}$ respectively. The cut-off points of BMI according to maximum sum of sensitivity and specificity for detecting hypertension, diabetes, and hypercholesterolemia in men were $25.7 \mathrm{~kg} / \mathrm{m}^{2}, 24.8 \mathrm{~kg} / \mathrm{m}^{2}$ and $24 \mathrm{~kg} / \mathrm{m}^{2}$ and in women were $26.9 \mathrm{~kg} / \mathrm{m}^{2}, 26.3 \mathrm{~kg} / \mathrm{m}^{2}$ and $26.1 \mathrm{~kg} / \mathrm{m}^{2}$ respectively.

Conclusions: This was a population-based study on a huge sample on the basis of a national survey. The Iranian BMI was different from the values suggested by the WHO. The waist circumference in Iranian women was higher than that in men.

Key words: cut-off point, body mass index, waist circumference.

\section{Introduction}

Obesity is one of the most serious health threats all over the world [1] and leads to chronic diseases such as cardiovascular disease (CVD), diabetes mellitus (DM), and dyslipidemia [2, 3]. The cut-off points of anthropometric indices such as body mass index (BMI) and waist circumference

\author{
Corresponding author: \\ Mohajeri-Tehrani \\ Mohammad Reza \\ Endocrinology \\ and Metabolism \\ Research Center \\ Tehran University \\ of Medical Sciences \\ $5^{\text {th }}$ Floor, Shariati Hospital \\ North Kargar Avenue \\ Tehran, Iran \\ Postal Code: 1411413137 \\ Phone: +98(21)88220037-38 \\ Fax: +98(21)88220052 \\ E-mail: \\ mrmohajeri@tums.ac.ir
}


(WC) are usually obtained by epidemiological studies conducted on Caucasians, especially Europeans; therefore, they are not necessarily representative of the indices in other ethnicities [4]. The values of these indices are varied in youth [5] and adults [68] with respect to race; for instance, the cut-off points of WC in studies in Oman, Iraq and Korea were $80 \mathrm{~cm}, 97 \mathrm{~cm}$ and $90 \mathrm{~cm}$ respectively in men and $84.5 \mathrm{~cm}, 99 \mathrm{~cm}$ and $85 \mathrm{~cm}$ respectively in women [6-8]. These cut-off points can also be dissimilar between the rural and urban populations of one region $[9,10]$. Obesity and its complications are more prevalent in the Middle East [11]. The World Health Organization (WHO) has defined the normal range of $\mathrm{BMI}$ as $18.5 \mathrm{~kg} / \mathrm{m}^{2}$ to $22 \mathrm{~kg} / \mathrm{m}^{2}$ in the Asian population [12]. Moreover, due to a lack of fresh data on the normal range of WC in the Middle East, the International Diabetes Federation (IDF) suggests that the normal range of Europeans, namely $94 \mathrm{~cm}$ in men and $80 \mathrm{~cm}$ in women, be applied for the definition of the normal WC in this region [13].

Given the paucity of information on the normal range of WC and BMI in Iran and the Middle East, at least in the adult population, we used data from the First Non-Communicable Disease (NCD) risk factors surveillance in Iran in 2005 to estimate the cutoff points of WC and BMI for detecting DM, hypercholesterolemia (HC), and hypertension (HTN).

\section{Material and methods}

The first national NCD risk factors surveillance was a cross-sectional study which was conducted in 2005, the detailed protocol of that is available elsewhere [14].

The samples were selected via the multi-stage cluster sampling method according to the WHO stepwise approach to NCD risk factors [15]. Each cluster contained 20 participants, half of each cluster being men.

In total, 89,440 participants aged between 15 and 64 years from the rural and non-institutionalized urban populations of 28 provinces of Iran were included. Our study was focused on 25 to 64-yearold individuals, so the sample size was reduced to 70,981.

A national committee was established to evaluate the protocols, guidelines, and tools for preventing any technical or ethical errors. The study was approved by the Ethics Committee of the Iranian Center for Disease Management and Control, and informed written and oral consent was obtained from the participants.

Height, weight, WC, and blood pressure were evaluated via physical examinations; fasting blood glucose (FBG) and cholesterol levels were assessed via laboratory tests. Data were gathered through a questionnaire based on the WHO's stepwise approach to NCD risk factors surveillance and filled in via face-to-face interviews by trained personnel. All the instruments were standardized before the examinations on a daily basis.

Height was measured using a portable inflexible bar while the participants were looking straight forward with their heels against the wall. Weight was measured with a daily calibrated analogue portable scale. During all the weight and height measurements, the individuals were in light clothing without shoes and socks. Waist circumference was measured using the standard constant tension tape, measuring the midaxillary line at the midpoint between the free head of the lower rib and the anterior superior iliac spine at the end of normal expiration [13]. Body mass index was estimated by dividing weight in kilograms by the square of the height in meters [11].

Blood pressure was measured with appropriatesized cuffs from the right arm and in the sitting position after 5 min' rest. The first Korotkoff sound indicated systolic blood pressure, and the disappearance of the sound signified diastolic blood pressure. The mean blood pressure after two measurements with 5-minute intervals was recorded. If the difference between systolic blood pressure and/or diastolic blood pressure in the two measurements was more than $10 \mathrm{~mm} \mathrm{Hg}$, the third measurement was recorded. Abnormal blood pressure was defined as a history of antihypertensive drug use or systolic blood pressure $\geq 140 \mathrm{~mm} \mathrm{Hg}$ or diastolic blood pressure $\geq 90 \mathrm{~mm} \mathrm{Hg}$ [16]. All the sphygmomanometers were calibrated daily by the Mercury Richter Sphygmomanometer.

Venous blood samples were obtained after 12 h' fasting and in the sitting position. Samples collected incorrectly or those obtained from non-fasting individuals were excluded.

Fasting blood glucose and cholesterol were assessed via enzymatic methods using the Pars Azmun (Pars Azmun Co., Tehran, Iran) Kit. The kits in all the laboratories bore the same serial and production permit numbers with the same expiration date. Fasting blood glucose was measured via the glucose oxidase/peroxidase-4 aminophenazonephenol (GOD-PAP) method, and cholesterol was assessed via the cholesterol oxidase/p-aminophenazone (CHD-PAP) method. Individuals with physician-diagnosed DM, as well as those with a history of oral hypoglycemic agents or insulin or with FBG > $126 \mathrm{mg} / \mathrm{dl}$ were categorized as diabetics [17]. Blood cholesterol level $\geq 200 \mathrm{mg} / \mathrm{dl}$ [18] or positive history of taking cholesterol-lowering agents was regarded as $\mathrm{HC}$.

\section{Statistical analysis}

The receiver operating characteristic (ROC) curves were employed to achieve the cut-off points of WC and BMI for detecting HTN, DM, and HC. The 
points with the maximum sum of sensitivity and specificity indicated the cut-off points. For analysis, SPSS software version 15 was applied.

\section{Results}

This study recruited 70,981 men and women between 25 and 64 years of age from 28 provinces of Iran. The study population comprised $50.5 \%$ men and $49.5 \%$ women, and the male-to-female ratio in each age group was approximately similar. Individuals from urban and rural areas accounted for $46.6 \%$ and $35.4 \%$ of the study population, respectively.

The mean $\mathrm{BMI}$ in the men and women was 25.1 $\pm 4.1 \mathrm{~kg} / \mathrm{m}^{2}$ and $27.2 \pm 5.0 \mathrm{~kg} / \mathrm{m}^{2}$, respectively. The mean WC was $88.7 \pm 12.3 \mathrm{~cm}$ in the men and 92.3 $\pm 13.8 \mathrm{~cm}$ in the women. The frequency of DM, HTN, and $\mathrm{HC}$ was $6.7 \%, 31.1 \%$, and $45.1 \%$ respectively in the men and $9.5 \%, 34.6 \%$, and $53.1 \%$ respectively in the women. In total, the frequency of DM, HTN, and $\mathrm{HC}$ was $8.1 \%, 32.8 \%$, and $49.3 \%$, respectively. The cut-off point of WC for detecting HTN with 63\% sensitivity (95\% Cl: $0.63-0.64)$ and $59 \%$ specificity ( $95 \%$ Cl: $0.59-0.60)$ was $89.7 \mathrm{~cm}$ in the men, whereas the cut-off point with $63 \%$ sensitivity $(95 \% \mathrm{Cl}$ : $0.62-0.63)$ and $60 \%$ specificity (95\% Cl: $0.60-0.61)$ was $93.9 \mathrm{~cm}$ in the women. The cut-off point of WC for detecting DM and $\mathrm{HC}$ was $89.4 \mathrm{~cm}$ and $88.2 \mathrm{~cm}$ respectively in the men and $96.2 \mathrm{~cm}$ and $90 \mathrm{~cm}$ respectively in the women. The cut-off points of $\mathrm{BMI}$ for detecting DM, HTN, and $\mathrm{HC}$ in the men and women are depicted in Table I.

All the cut-off points of WC and BMI in association with the above-mentioned complications were higher in the women than in the men. The cut-off points of BMI in both genders and the cut-off point of WC in the men for detecting HTN were higher than those for DM, and the cut-off points for detecting DM were higher than those for $\mathrm{HC}$. On the other hand, in the women, the cut-off point for detecting DM was higher than that for HTN and the cut-off point for detecting HTN was higher than that for HC (Table I).

\section{Discussion}

In other studies the cut-off points of WC and BMI are different from ours (Tables II-III). For instance, the WHO has defined normal WC as $102 \mathrm{~cm}$ in men and $88 \mathrm{~cm}$ in women [1] and normal BMI in Asians as $18.5 \mathrm{~kg} / \mathrm{m}^{2}$ to $22 \mathrm{~kg} / \mathrm{m}^{2}$ due to a higher prevalence of DM in lower BMI ranges in this region [12] However, the IDF has suggested that the normal ranges of Europeans, namely $94 \mathrm{~cm}$ in men and $80 \mathrm{~cm}$ in women, be applied for a definition of normal WC in the Middle East [13]. According to a study in the capital of Iran (Tehran), normal WC in men and women was defined as $91.5 \mathrm{~cm}$ and $85.5 \mathrm{~cm}$, respectively [18]. This study was conducted in 2008 on 2,752 participants aged between 18 and 80 from 4 geographical regions of Tehran; the sensitivity and specificity of the cut-off points were compared with the ranges recommended by the IDF and the Adult Treatment Panel III (ATP III). The sensitivity of WC in men and women was $77 \%$ and $86 \%$, respectively; and the specificity was $58 \%$ and $50 \%$, respectively. The sensitivity of WC in men was higher than that suggested by the IDF and it was lower in women. Furthermore, specificity in men was lower than that suggested by the IDF but it was higher in women [18]. In comparison with the ATP III, which defines normal WC as $102 \mathrm{~cm}$ in men and $88 \mathrm{~cm}$ in women [19], in both genders sensitivity

Table I. Cut-off points of waist circumference and body mass index for detecting diabetes, hypercholesterolemia, and hypertension in 25 to 64-year-old Iranians according to the First Non-Communicable Disease Survey in Iran in 2005

\begin{tabular}{|c|c|c|c|c|c|}
\hline Disease & Gender & $\begin{array}{l}\text { Anthropometric } \\
\text { indices }\end{array}$ & $\begin{array}{l}\text { Cut-off } \\
\text { point }\end{array}$ & $\begin{array}{l}\text { Sensitivity } \\
(95 \% \mathrm{Cl})\end{array}$ & $\begin{array}{l}\text { Specificity } \\
(95 \% \mathrm{Cl})\end{array}$ \\
\hline \multirow{4}{*}{ Hypertension } & \multirow{2}{*}{ Male } & BMI & 25.7 & $0.55(0.54-0.55)$ & $0.64(0.64-0.65)$ \\
\hline & & WC & 89.7 & $0.63(0.63-0.64)$ & $0.59(0.59-0.60)$ \\
\hline & \multirow{2}{*}{ Female } & BMI & 26.9 & $0.62(0.62-0.62)$ & $0.57(0.56-0.57)$ \\
\hline & & WC & 93.9 & $0.63(0.62-0.63)$ & $0.60(0.60-0.61)$ \\
\hline \multirow{4}{*}{ Diabetes } & \multirow{2}{*}{ Male } & BMI & 24.8 & $0.69(0.68-0.69)$ & $0.50(0.50-0.51)$ \\
\hline & & WC & 89.4 & $0.71(0.70-0.71)$ & $0.53(0.53-0.54)$ \\
\hline & \multirow{2}{*}{ Female } & BMI & 26.3 & $0.71(0.71-0.71)$ & $0.46(0.46-0.47)$ \\
\hline & & WC & 96.2 & $0.61(0.61-0.62)$ & $0.62(0.62-0.63)$ \\
\hline \multirow{4}{*}{ Hypercholesterolemia } & \multirow{2}{*}{ Male } & BMI & 24.0 & $0.69(0.69-0.70)$ & $0.49(0.49-0.50)$ \\
\hline & & WC & 88.2 & $0.62(0.62-0.63)$ & $0.57(0.57-0.58)$ \\
\hline & \multirow{2}{*}{ Female } & BMI & 26.1 & $0.64(0.64-0.65)$ & $0.51(0.50-0.51)$ \\
\hline & & WC & 90.0 & $0.66(0.65-0.66)$ & $0.53(0.52-0.53)$ \\
\hline
\end{tabular}

$B M I-$ body mass index $\left[\mathrm{kg} / \mathrm{m}^{2}\right], W C$ - waist circumference $[\mathrm{cm}]$ 
Cut-off points of waist circumference and body mass index for detecting diabetes, hypercholesterolemia and hypertension according to National Non-Communicable Disease Risk Factors Surveillance in Iran

Table II. Cut-off points of waist circumference in different studies and their comparison with our results

\begin{tabular}{|lcc|}
\hline Study name [reference] & Cut-off points of waist circumference [cm] \\
\cline { 2 - 3 } & Men & Women \\
\hline Our study (for detecting hypertension) & 89.7 & 93.9 \\
\hline Our study (for detecting diabetes) & 89.4 & 96.2 \\
\hline Our study (for detecting hypercholesterolemia) & 88.2 & 90 \\
\hline Iran (for detecting metabolic syndrome) [20] & 89 & 91 \\
\hline Iran (for detecting cardiovascular disease) [22] & 94.5 & 94.5 \\
\hline Tehran (Iran) (for detecting metabolic syndrome risk factors) [19] & 91.5 & 85.5 \\
\hline International Diabetes Federation (normal ranges) [13] & 94 & 80 \\
\hline World Health Organization (normal ranges) [1] & 102 & 88 \\
\hline Adult Treatment Panel III (normal ranges) [18, 19] & 102 & 88 \\
\hline Korea (for detecting metabolic syndrome) [24] & 83 & 76 \\
\hline Korea (for detecting diabetes) [24] & 85 & 77 \\
\hline Korea (for detecting ischemic heart disease ) [24] & 87 & 74 \\
\hline Korea (for detecting hypertension) [24] & 84 & 77 \\
\hline Korea (for detecting central obesity) [7] & 85 & 80 \\
\hline Japan (for detecting central obesity) [25] & 89.8 & 82.3 \\
\hline China (for detecting metabolic syndrome) [26] & 90 & 85 \\
\hline China (for detecting central obesity) [27] & 85 & 80 \\
\hline Oman (for detecting cardiovascular disease risk factors) [6] & 80 & 84.5 \\
\hline Iraq (for detecting metabolic syndrome) [8] & 97 & 99 \\
\hline Tunisia (for detecting central obesity by detecting cardiovascular \\
disease and diabetes) [23] & 85 & 80 \\
\hline Asian Indian (normal ranges) [28] & & 85 \\
\hline
\end{tabular}

Table III. Cut-off points of body mass index in different studies and their comparison with our results

\begin{tabular}{|lcc|}
\hline Study name [reference] & \multicolumn{2}{c|}{ Body mass index [kg/m²] } \\
\cline { 2 - 3 } & Men & Women \\
\hline Our study (for detecting hypertension) & 25.7 & 26.9 \\
\hline Our study (for detecting diabetes) & 24.8 & 26.3 \\
\hline Our study (for detecting hypercholesterolemia) & 24 & 26.1 \\
\hline World Health Organization (normal ranges in Asians) [12] & 18.5 to 22 & 18.5 to 22 \\
\hline Asian study (for detecting obesity) [29] & 27 & 25 \\
\hline Iran (for detecting cardiovascular disease) [22] & 26.9 & 29.1 \\
\hline China (for detecting chronic disease risk factors by detecting overweight) [27]* & 24 & 24 \\
\hline China (for detecting chronic disease risk factors by detecting obesity) [27] ${ }^{*}$ & 28 & 28 \\
\hline Oman (for detecting cardiovascular disease risk factors) [6] & 23.2 & 26.8 \\
\hline Tunisia (for detecting cardiovascular disease) [23] & 24 & 27 \\
\hline Indian Asian (normal ranges) [28] & 23 & 23 \\
\hline
\end{tabular}

*The gender difference is not mentioned in the main article

was higher and specificity was lower [18]. In this study, the value of WC in women was lower than that in men, which was not consistent with our results. One possible explanation is the fact that women in Tehran work outside of their houses more than other Iranian women.

In the NCD Risk Factors Surveillance, which was carried out in 2007 in Iran, 3,027 men and women 
from 30 provinces participated; the best cut-off point of WC for detecting the metabolic syndrome was $89 \mathrm{~cm}$ in men and $91 \mathrm{~cm}$ in women [20]. The WC was higher in women and it was in line with our results. In Iranian culture, the number of women with a paid job outside of their houses is lower than that of men. Additionally, lack of physical activity is seen in $70 \%$ to $80 \%$ of the Iranian population and it is thus one of the general health problems [21].

Hadaegh et al. conducted a study in Iran over a period of 7.6 years on 3,620 men and women over 40 years old with no history of CVD to determine the anthropometric values with respect to the incidence of CVD. The cut-off point of BMI according to the ROC curves and maximum sensitivity and specificity was $26.95 \mathrm{~kg} / \mathrm{m}^{2}$ in men and $29.19 \mathrm{~kg} / \mathrm{m}^{2}$ in women. The cut-off point of WC for detecting CVD according to maximum sensitivity and specificity in both genders was $94.5 \mathrm{~cm}$ [22]. Whereas the said study was a longitudinal population-based study, ours was a cross-sectional one conducted on a huge sample $(79,611$ participants). The suggested value for the cut-off point of WC in Oman for detecting CVD risk factors defined by at least 2 of 3 risk factors (hyperglycemia, HTN, and dyslipidemia) was $80 \mathrm{~cm}$ in men and $84.5 \mathrm{~cm}$ in women [6]. In Iraq, the cut-off point according to maximum sensitivity and specificity for detecting the metabolic syndrome was $97 \mathrm{~cm}$ in men and $99 \mathrm{~cm}$ in women. Moreover, the sensitivity and specificity of applying these values were $70 \%$ and $50 \%$ respectively in men and $70 \%$ and $45 \%$ respectively in women [8]. In Tunisia, the cut-off point according to the ROC curves for diagnosing central obesity and for detecting CVD risk factors and DM in both genders was $85 \mathrm{~cm}$. The WC value, which was $85 \mathrm{~cm}$ in men and $79 \mathrm{~cm}$ in women, had more than $90 \%$ sensitivity and more than $83 \%$ specificity for detecting individuals with $\mathrm{BMI} \geq 25 \mathrm{~kg} / \mathrm{m}^{2}$ [23].

In Korea, men with $90 \mathrm{~cm}$ and women with $86.5 \mathrm{~cm}$ WC were in the $80^{\text {th }}$ percentile of WC, and the cut-off point for detecting central obesity was $85 \mathrm{~cm}$ in men and $80 \mathrm{~cm}$ in women [7]. In another study in Korea, the cut-off point of WC according to maximum sensitivity and specificity for detecting ischemic myocardial diseases in men and women was $87 \mathrm{~cm}$ and $74 \mathrm{~cm}$ respectively and for detecting HTN, DM, and risk factors of the metabolic syndrome was $84 \mathrm{~cm}, 85 \mathrm{~cm}$, and $83 \mathrm{~cm}$ in men and $77 \mathrm{~cm}, 77 \mathrm{~cm}$, and $76 \mathrm{~cm}$ in women, respectively [24]. In Japan, the best cut-off point by the ROC curves for detecting central obesity in men was higher than that in women $(89.8 \mathrm{~cm}$ vs. $82.3 \mathrm{~cm})$ [25]. In China, this value according to the ROC curves for detecting the metabolic syndrome was $90 \mathrm{~cm}$ in men and $85 \mathrm{~cm}$ in women [26]. In another study in China, the best cut-off point for detecting central obesity was
$85 \mathrm{~cm}$ in men and $80 \mathrm{~cm}$ in women [27]. The normal WC value in the Asian Indian population was $85 \mathrm{~cm}$ in men and $80 \mathrm{~cm}$ in women [28].

In our study, WC was higher in the women than in the men and it was in line with the Oman and Iraq studies [6, 8] as well as with the Iranian Survey in 2007 [20] but it did not agree with the Tehran [19], China [26, 27], Japan [25], Korea [7, 24], and Asian Indian studies [28] or with the values recommended by the IDF [13], ATP III [18, 19], and WHO [1] (Table II). One possible explanation is that most Iranian women are housewives and they have a lesser role in social activities. But maybe the women in these countries work outside of their houses and these life habit changes and outdoor jobs are also seen nowadays in women who live in the capital city of Iran.

A comparison of the WC values between our females and those in other studies $[1,6,7,13,18$, $19,23-28$ ] demonstrates that only WC in Iraq is higher than that in our study [8]. The WC values in the Hadaegh et al. study were higher than our values for detecting HTN and HC but lower for detecting DM. Their values, however, were approximately in line with ours [22]. The values reported in the Iranian survey in 2007 [20] for detecting HC were higher than our value but lower for detecting HTN and DM (Table II).

A comparison of WC between men in our study and those in other studies in the existing literature shows that WC amongst our males was higher than that in the studies in Oman [6], Korea [24], Tunisia [23], China [27], and Asian Indians [28] but lower than not only the values in the studies in Iraq [8], Korea [7], China [26], Tehran [19], and the Hadaegh et al. study [22] but also the values recommended by the WHO, IDF, and ATP III [1, 13, 18, 19].

The WC in our study for detecting HTN was higher than the Japanese values, but it was lower for detecting DM and HC [25]. Nonetheless, our values are more or less along the lines of the ones reported in Japan [25], Korea [7], and China [26].

The WC values for detecting HTN and DM in our men were higher and for detecting $\mathrm{HC}$ were lower than the WC value for detecting the metabolic syndrome in the Iranian survey in 2007 [20]. Still, our values are almost concordant with those in the said survey.

By comparing the variety of WC cut-off points obtained from different studies, it could be seen that the cut-off points for detecting chronic conditions such as DM, HTN and HC differ according to gender and ethnicity. Moreover, each of these conditions for detecting needs its own specific values.

Since the cut-off points of WC for detecting $\mathrm{HC}$ in both genders are less than the values for detecting DM and HTN, the recommended cut-off points of WC for preventing all three complications (DM, 
$\mathrm{HTN}$ and $\mathrm{HC}$ ) in men and women are less than $88.2 \mathrm{~cm}$ and $90 \mathrm{~cm}$ respectively (Table $\mathrm{II}$ ).

In Tunisia, BMI for detecting CVD risk factors was $24 \mathrm{~kg} / \mathrm{m}^{2}$ in men and $27 \mathrm{~kg} / \mathrm{m}^{2}$ in women [23]. In Oman, the optimal ranges of $\mathrm{BMI}$ in men and women (for detecting CVD) were $23.2 \mathrm{~kg} / \mathrm{m}^{2}$ and $26.8 \mathrm{~kg} / \mathrm{m}^{2}$, respectively [6]. In these studies, like ours, the range of BMI in women was higher than that in men; but in Indian Asians, the normal cut-off point of BMI in both genders was $23 \mathrm{~kg} / \mathrm{m}^{2}$ [28] (Table III).

In our study, the reference range of BMI for detecting $\mathrm{HC}$ in men was $24 \mathrm{~kg} / \mathrm{m}^{2}$, which is in line with the range of BMI for detecting CVD in Tunisian men. Nevertheless, reference ranges for detecting HTN and DM in our study were higher than those in Tunisian men. On the other hand, in our women, the reference range of BMI for detecting HTN was approximately the same as the reference range of $\mathrm{BMI}$ for detecting CVD in Tunisian women, and the values in our study for detecting DM and $\mathrm{HC}$ were lower than theirs [23].

In our study, the reference range of $\mathrm{BMI}$ in the men was higher than that in Oman and Indian Asian men $[6,28]$ and also it was higher than that recommended by the $\mathrm{WHO}$ [12]. The reference range of BMI for detecting HTN in our women was approximately the same as that in the Oman study, but the ranges for detecting $\mathrm{DM}$ and $\mathrm{HC}$ were lower in our women than in the Oman women [6] and were higher than the ranges in Indian Asians and values recommended by the WHO $[12,28]$ (Table III).

Since the cut-off points of BMI for detecting HC in both genders are less than the values for detecting DM and HTN, the recommended cut-off points of BMI for preventing all three complications (DM, $\mathrm{HTN}$ and $\mathrm{HC}$ ) in men and women are $24 \mathrm{~kg} / \mathrm{m}^{2}$ and $26.1 \mathrm{~kg} / \mathrm{m}^{2}$ respectively (Table III).

In one Asian study, by reducing the $\mathrm{BMI}$ ranges for detecting obesity from $30 \mathrm{~kg} / \mathrm{m}^{2}$ to $27 \mathrm{~kg} / \mathrm{m}^{2}$ in men and $25 \mathrm{~kg} / \mathrm{m}^{2}$ in women, sensitivity in men rose from $6.7 \%$ to $46.7 \%$ and sensitivity in women rose from $13.4 \%$ to $60.8 \%$ [29]. In China, the reference range of $\mathrm{BMI}$ for defining overweight with best sensitivity and specificity for detecting chronic disease risk factors (hypertension, diabetes, dyslipidemia) was $24 \mathrm{~kg} / \mathrm{m}^{2}$ and the cut-off point of BMI for defining obesity with $90 \%$ specificity was $28 \mathrm{~kg} / \mathrm{m}^{2}$ [27] (Table III).

The South Asian population, the Chinese, and the aboriginal Canadians in comparison with Europeans are believed to have lower BMI but approximately the same lipid and glucose levels. Consequently, the cut-off point for the definition of obesity with respect to risk factors associated with glucose and lipid levels in Europeans is approximately $6 \mathrm{~kg} / \mathrm{m}^{2}$ higher than in non-Europeans [4].

Comparison of the cut-off points of BMI reported from different studies indicates that these values for detecting DM, HTN and WC differ according to gen- der and race. Moreover, each of these conditions for detection needs its own specific cut-off points.

In conclusion, this study was conducted according to the First Non-Communicable Disease Survey in Iran in 2005 and recruited a huge sample size. Our results show that the normal ranges of WC and $\mathrm{BMI}$ in Iranians were different from those of some other Asians as reported by the WHO [12] or the IDF [13]; approximately the same as the values of some Asian [6, 27] and African [23] populations; and similar to the ranges in the Hadaegh et al. study in Iran [22]. Our purpose in comparing the different cut-off points from different studies was to show the fact that these values vary according to gender and ethnicity. They are also different for detecting diabetes, hypertension and hypercholesterolemia.

The recommended cut-off points of BMI and WC for preventing all three complications (DM, HTN and $\mathrm{HC}$ ) in men are $24 \mathrm{~kg} / \mathrm{m}^{2}$ and $88.2 \mathrm{~cm}$ respectively and in women are $26.1 \mathrm{~kg} / \mathrm{m}^{2}$ and $90 \mathrm{~cm}$ respectively. The WC range in our Iranian females was higher than that in our men, which agrees with the results from some other studies carried out in the Middle East $[6,8]$ and which may be due to the lesser role that women play in social activities in this part of the world. Another possible contributing factor is urbanization and the concomitant lifestyle changes and lower physical and social activities [1, 30-32]. It is also worth noting that WC was higher in the Iranian women than in the women from some other Asian countries [7, 24-28].

Given the higher BMI and WC in the women in our study and the direct link between obesity and CVD, DM, and dyslipidemia [2, 3], it is prudent that the causes of this phenomenon and their effect on women's health be fully investigated and the significance of losing weight and lifestyle modification be emphasized.

Some of the limitations of this study are the non-measurement of other lipid levels such as lowdensity lipoprotein (LDL), high-density lipoprotein (HDL) and triglycerides and also non-measurement of other metabolic syndrome criteria [13], which were not measured in the national survey in 2005. The lipid profiles have been measured in the Iranian national survey in 2007 [20, 33]. On the other hand, our study was cross-sectional and was thus unable to follow the complications. It is, therefore, recommended that follow-up studies be conducted to determine the accurate cut-off points of anthropometric indices.

\section{Acknowledgments}

We drew upon data from the First Iranian National NCD Risk Factors Surveillance; therefore we hereby wish to thank the Non Communicable Disease Management office of the Iranian Ministry of Health and Medical Education. Many thanks are also due 
to the Endocrinology and Metabolism Research Center of Tehran University of Medical Sciences.

\section{References}

1. WHO Technical Report Series 916 .Diet, nutrition and the prevention of chronic diseases. Report of a Joint WHO/FAO Expert Consultation. World Health Organization. Geneva. 2003. FAO Corporate Document Repository. http:// www.fao.org/DOCREP/005/AC911E/AC911E00.HTM. Accessed 26 July 2010.

2. Seidell JC, Kahn HS, Williamson DF, Lissner L, Valdez R. Report from a centers for disease control and prevention workshop on use of adult anthropometry for public health and priming health care. Am J Clin Nutr 2001; 73: 123-6.

3. Boyd GS, Koenigsberg J, Falkner B, Gidding S, Hassink S. Effect of obesity and high blood pressure on plasma lipid levels in children and adolescents. Pediatrics 2005; 116: 442-6.

4. Razak F, Anand SS, Shannon H, et al. Defining obesity cut points in a multiethnic population. Circulation 2007; 115 2111-8.

5. Kelishadi R, Schwandt P, Haas GM, Hosseini M, Mirmoghtadaee $P$. Reference curves of anthropometric indices and serum lipid profiles in representative samples of Asian and European children. Arch Med Sci 2008; 4: 329-35.

6. Al-Lawati JA, Jousilahti P. Body mass index, waist circumference and waist-to-hip ratio cut-off points for categorization of obesity among Omani Arabs. Public Health Nutr 2007; 11: 102-8.

7. Lee SY, Park HS, Kim DJ, et al. Appropriate waist circumference cutoff points for central obesity in Korean adults. Diabetes Res Clin Pract 2007; 75: 72-80.

8. Mansour AA, Al-Hassan AA, Al-Jazairi MI. Cut-off values for waist circumference in rural Iraqi adults for the diagnosis of metabolic syndrome. Rural Remote Health 2007; 7: 765 .

9. Deurenberg P, Deurenberg YM, Guricci S. Asians are different from Caucasians and from each other in their body mass index/body fat percent relationship. Obes Rev 2002; 3: 141-6.

10. The International Association for the Study of Obesity. Obesity in Asia Collaboration (OAC). Ethnic comparison of obesity in the Asia-Pacific region: protocol for a collaborative overview of cross sectional studies. Obes Rev 2005; 6: 193-8.

11. WHO expert consultation. Appropriate body mass index for Asian population and its implications for policy and intervention strategies. Lancet 2004; 363: 157-63.

12. World Health Organization. Obesity: preventing and managing the global epidemic. World Health Organ Tech Rep Ser 2000; 894: 1-253.

13. Alberti KG, Zimmet P, Shaw J. Metabolic syndrome: a new world-wide definition. A consensus statement from the International Diabetes Federation. Diabet Med 2006; 23: 469-80.

14. Alikhani S, Delavari A, Alaedini F, Kelishadi R, Rohbani S, Safaei A. A province-based surveillance system for the risk factors of non-communicable diseases: a prototype for integration of risk factor surveillance into primary healthcare systems of developing countries. Public Health 2009; 123: 358-64.

15. World Health Organization. WHO stepwise approach to Surveillance. Geneva. 2004. World Health Organization. http://www.who.int/ncd_surveillance/steps/en. Accessed: 21 August 2004.
16. Chobanian AV, Bakris GL, Black HR, et al. The National High Blood Pressure Education Program Coordinating Committee. Seventh report of the Joint National Committee on Prevention, Detection, Evaluation, and Treatment of High Blood Pressure. Hypertension 2003; 42: 1206-52.

17. American Diabetes Association. Diagnosis and Classification of Diabetes Mellitus (position statement). Diabetes Care 2005; 28 (Suppl. 1): S37-42.

18. Adult Treatment Panel III. Third Report of the National Cholesterol Education Program (NCEP) expert panel on detection, evaluation, and treatment of high blood cholesterol in adults (Adult Treatment Panel III). Final report. Circulation 2002; 106: 3143-421.

19. Esteghamati A, Ashraf H, Rashidi A, Meysamie A. Waist circumference cut-off points for the diagnosis of metabolic syndrome in Iranian adults. Diabetes Res Clin Pract 2008; 82: 104-7.

20. Delavari A, Forouzanfar MH, Alikhani S, Sharifian A, Kelishadi R. First nationwide study of the prevalence of the metabolic syndrome and optimal cutoff points of waist circumference in the Middle East: the national survey of risk factors for noncommunicable diseases of Iran. Diabetes Care 2009; 32: 1092-7.

21. Sheikholeslam R, Mohamad A, Mohammad K, Vaseghi S. Non communicable disease risk factors in Iran. Asia Pac J Clin Nutr 2004; 13 (Suppl): S100.

22. Hadaegh F, Zabetian A, Sarbakhsh P, Khalili D, James WPT, Azizi F. Appropriate cutoff values of anthropometric variables to predict cardiovascular outcomes: 7.6 years follow-up in an Iranian population. Int J Obes (Lond) 2009; 33: 1437-45.

23. Bouguerra R, Alberti H, Smida H, et al. Waist circumference cut-off points for identification of abdominal obesity among the Tunisian adult population. Diabetes Obes Metab 2007; 9: 859-68.

24. Kim HK, Kim CH, Park JY, Lee KU. Lower waist-circumference cutoff point for the assessment of cardiometabolic risk in Koreans. Diabetes Res Clin Pract 2009; 85: 35-9.

25. Oka R, Kobayashi J, Yagi K, et al. Reassessment of the cutoff values of waist circumference and visceral fat area for identifying Japanese subjects at risk for the metabolic syndrome. Diabetes Res Clin Pract 2008; 79: 474-81.

26. Bao Y, Lu J, Wang C, et al. Optimal waist circumference cutoffs for abdominal obesity in Chinese. Atherosclerosis 2008; 201: 378-84

27. Bei-Fan Z; Cooperative Meta-Analysis Group of Working Group on Obesity in China. Predictive values of body mass index and waist circumference for risk factors of certain related diseases in Chinese adults: study on optimal cutoff points of body mass index and waist circumference in Chinese adults. Asia Pac J Clin Nutr 2002; 11 Suppl 8: S685-93.

28. Snehalatha C, Viswanathan V, Ramachandran A. Cutoff values for normal anthropometric variables in asian Indian adults. Diabetes Care 2003; 26: 1380-4.

29. Goh VHH, Tain CF, Tong TYY, Mok HPP, Wong MT. Are BMI and other anthropometric measures appropriate as indices for obesity? A study in an Asian population. J Lipid Res 2004; 45: 1892-8.

30. Malekzadeh R, Mohammadnejad M, Merat Sh, Pourshams A, Etemadi A. Obesity pandemic: an Iranian perspective. Arch Iran Med 2005; 8: 1-7.

31. Howarth NC, Murphy SP, Wilkens LR, Hankin JH, Kolonel LN. Dietary energy density is associated with overweight status among 5 ethnic groups in the multiethnic cohort study. J Nutr 2006; 136: 2243-8. 
Cut-off points of waist circumference and body mass index for detecting diabetes, hypercholesterolemia and hypertension according to National Non-Communicable Disease Risk Factors Surveillance in Iran

32. Eckel RH, Krauss RM. American Heart Association call to action: obesity as a major risk factor for coronary heart disease. Circulation 1998; 97: 2099-100.

33. Delavari A, Kelishadi R, Forouzanfar MH, Safaei A, Birjandi F, Alikhani S. The first cut-off points for generalized and abdominal obesity in predicting lipid disorders in a nationally representative population in the Middle East: The National Survey of Risk Factors for Non-Communicable Diseases of Iran. Arch Med Sci 2009; 5: 542-9. 